\title{
"Semillas de un Nobel" como estrategia para mejorar la redacción de estudiantes universitarios
}

"Seeds of a Nobel" as a strategy to improve university students writing skills 


\title{
"Semillas de un Nobel" como estrategia para mejorar la redacción de estudiantes universitarios ${ }^{1}$ \\ "Seeds of a Nobel" as a strategy to improve university students writing skills
}

\section{Gonzalo Rojas Reyes² ${ }^{2}$ Fabio Estupiñán Gómez ${ }^{3}$, Juan Miguel Quintero Saldaña ${ }^{4}$, Hermes Julian Mora Santos ${ }^{5}$}

Artículo recibido en agosto 13 de 2019; artículo aceptado en noviembre 18 de 2019

\begin{abstract}
Este artículo puede compartirse bajo la licencia Licencia Creative, Commons Atribución-No Comercial-Compartir Igual 4.0 Internacional y se referencia usando el siguiente formato: Rojas, G., Estupiñan, F., Quintero, J. M. \& Mora, H. J. (2020). "Semillas de un Nobel" como estrategia para mejorar la redacción de estudiantes universitarios. I+D Revista de Investigaciones, 15 (1), $49-56$.
\end{abstract}

DOI: https://doi.org/10.33304/revinv.v15n1-2020005

\section{Resumen}

Este artículo es resultado de la puesta en práctica de la estrategia "Semillas de un Nobel"(SDUN), que fue diseñada para mejorar la redacción de estudiantes universitarios. Se trabajó con una muestra de 27 participantes. La metodología empleada fue de enfoque cualitativo. El proceso se desarrolló en tres fases: diseño, implementación y evaluación de la estrategia. Se utilizó como instrumento de recolección, diagnóstico, análisis y evaluación de información la modalidad conferencia-laboratorio, así como la aplicación de una entrevista semiestructurada. Entre los resultados se encontró que la aplicación de SDUN ayuda a los estudiantes a mejorar considerablemente sus competencias para redactar textos académicos. Se observó una mejora del $33 \%$ en redacción, desde las dimensiones coherencia, sencillez y ortografía, y un $60 \%$, desde las dimensiones redacción y construcción de microrrelatos.

Palabras clave: Estrategia didáctica, microrrelato, redacción, semillas de un nobel.

\footnotetext{
${ }^{1}$ Artículo de investigación, de paradigma cualitativo y enfoque hermenéutico, resultado de un proyecto de investigación culminado, perteneciente al área de ciencias sociales y humanísticas, subárea técnicas de expresión oral y escrita, desarrollado en el Grupo de Investigación SDUN, financiado por la Universidad de Investigación y Desarrollo, sede San Gil (Colombia). Dirección: carr. 9 n. ${ }^{\circ}$ 10-40. PBX: 7242224. Fecha de inicio: 6 de julio de 2018. Fecha de terminación: 30 de noviembre de 2019.

${ }^{2}$ Licenciado en Filosofía y Letras. Doctor en Ciencias Sociales. Docente investigador, Universidad de Investigación y Desarrollo, sede San Gil (Colombia). Dirección: carr. 9 n. ${ }^{\circ}$ 10-40. PBX: 7242224. ORCID ID: https://orcid.org/0000-0001-9376-7152. Correo electrónico institucional: grojas4@ udi.edu.co.

${ }^{3}$ Microbiólogo. Magíster en Educación, con mención en Currículum, Doctorando en Educación. Docente investigador y Coordinador de Investigaciones, Universidad de Investigación y Desarrollo, sede San Gil (Colombia). Dirección: carr. 9 n. ${ }^{\circ}$ 10-40. PBX: 7242224. ORCID ID: https:// orcid.org/0000-0002-3185-2105. Correo electrónico institucional: investigacionessg@udi.edu.co.

${ }^{4}$ Administrador de Empresas. Magíster en Administración. Magíster en Gestión de la Tecnología Educativa. Doctorando en Administración Gerencial. Docente investigador, Universidad de Investigación y Desarrollo, sede San Gil (Colombia). Dirección: carr. 9 n. ${ }^{\circ} 10-40$. PBX: 7242224. Correo electrónico institucional. ORCID ID: https://orcid.org/0000-0001-9071-7870. Correo electrónico institucional: jquintero8@udi.edu.co.

${ }^{5}$ Licenciado en Teología. Magíster en Educación con mención en Pedagogía. Mentor del proyecto Semillas de un Nobel (SDUN). Docente medio tiempo, Universidad de Investigación y Desarrollo, sede San Gil (Colombia). Dirección: carr. 9 n. ${ }^{\circ}$ 10-40. PBX: 7242224. ORCID ID: https://orcid. org/0000-0003-4546-352X. Correo electrónico institucional: hmora1@udi.edu.co.
} 


\begin{abstract}
This article is the result of the implementation of the "Seeds of a Nobel" (SDUN) strategy, which was designed to improve university students writing skills. A qualitative approach methodology was used, with a sample of 27 participants. The process was developed in three phases: design, implementation, and evaluation of the strategy. The conferencelaboratory modality was used as an instrument for collecting, diagnosing, analyzing and evaluating information, as well as for the application of a semi-structured interview. Among the results, it was found that the SDUN application helps students to significantly improve their academic writing skills. There was an improvement of $33 \%$ in dimensions such as coherence, simplicity and spelling, and of $60 \%$ in the writing and development of flash fiction.
\end{abstract}

Keywords: Teaching strategy, flash fiction, writing, seeds of a nobel.

\section{Introducción}

Este artículo es el resultado de la puesta en práctica de la estrategia "Semillas de un Nobel" (SDUN), diseñada para mejorar las capacidades de redacción en estudiantes universitarios. Para que una estrategia didáctica surta efecto en los procesos de enseñanza y aprendizaje, es necesario que sintonice con los planes y secuencias previstos, para alcanzar metas específicas acordes con el currículo (Schemeck, 1988).

¿Qué entendemos por redacción de un texto? Para Salazar (1999), es una actividad comunicativa de primer orden, en la que la persona exterioriza mediante grafemas aquello que construye en su pensamiento. Castelló, Pozo y Pérez (2009) afirman: "En la Universidad, la actividad escritora debe ser un ejercicio de creación y no tanto de transcripción". Lo dicho se complementa con el aporte de Pérez-Abril y Rodríguez (2013), que sostienen que la lectura y la escritura son factores que permiten visualizar los alcances del desarrollo cognitivo de la población de un país y su impacto en su desarrollo. Para el caso colombiano, aún queda abundante trabajo por realizar; faltan más estrategias que conduzcan a incitar, facilitar y fomentar la redacción (Carrasco, Encinas, Castro \& López, 2013). El Centro Regional para el Fomento del Libro en América Latina, el Caribe, España y Portugal (CERLALC, 2007) intenta mostrar la complementariedad de los actos de escribir y redactar: el primero, mediante las letras, explora, experimenta e imagina posibilidades; y el segundo concatena las ideas con los textos, lo que implica una secuencia de fases: preescritura (activación deseo), escritura (plasmación) y reescritura (edición). Dicho aporte se complementa con el aporte de Timbal (1993), que explicita que "redactar es una habilidad cognitiva compleja que se aprende, por lo cual requiere formación y entrenamiento".

Los estudiantes universitarios presentan serios problemas de expresión escrita, específicamente en lo que concierne a cohesión, coherencia e informatividad (Sánchez, 2005). Según Fregoso (2008), la falencia en cuanto a este tipo de comunicación en estudiantes universitarios es latente, y no solo en pregrado, sino también en posgrado. Rupturas ortográficas, expresión no fluida y falta de fundamentación e interrelación con otros textos son algunas de las principales dificultades. Ante este panorama, se requieren diversas alternativas que cualifiquen el ejercicio de redacción, ya que en el contexto universitario es prerrequisito a la hora de hacer investigación (Sabaj, 2009).

Son diversas las estrategias que se han empleado con el fin de mejorar la redacción en estudiantes universitarios. Las que se han traído aquí a colación son algunas de las más relevantes. Espinosa, Zambrano, Martínez y Suárez (2005), en un proyecto realizado en México, afirman que el fomentar cursos académicos específicos y establecidos para fortalecer la redacción en estudiantes universitarios causa impacto positivo. Arrieta y Meza (2005), como resultado de una investigación hecha en Venezuela, sostienen que la técnica de diagnóstico, escritura, reescritura, lectura en voz alta y discusión de contenidos funciona, pero que, para que esta alcance estándares más altos, se requieren más tiempo y más sesiones. Ocampo (2016), de nacionalidad colombiana, con su técnica denominada "La ensalada de cuentos", con base en sus fases de composición, revisión y socialización, concluyó que es una gran herramienta a la hora de fomentar la redacción de textos, específicamente lo que atañe a microestructura, macroestructura y superestructura.

Los tres autores mencionados coinciden en que tanto el tiempo dedicado a dichas actividades como el reducido número de sesiones son insuficientes y limitan el alcance de mayores porcentajes en cuanto a idoneidad en redacción.

Ahora bien, acudir a las artes literarias, en este caso específico al microrrelato, favorece sobremanera el proceso de estructurar y expresar el conocimiento. Pero ¿qué es el microrrelato? Para Álvarez y Mosquera (2014), es un texto breve, un ejercicio de condensación; para Zaid (2004), la representación gráfica de un escrito; $y$, para García (2012), es una frase o sentencia plasmada con maestría. Para Castañeda (2011), es un texto que se adhiere 
sensualmente a lo esencial y permite la experimentación; y para Lagmanovich (2008), un escrito sorpresivo, fugaz y fecundo que alude a otras narrativas (transtextualidad) y se caracteriza por su carácter metafórico.

En SDUN, el microrrelato, gracias a su brevedad, uso de metáforas y transtextualidad (Bustamante, 2012), resulta ser una excelente herramienta para mejorar la redacción desde la óptica argumentativo-científica. Esta es una de las conclusiones a la que llegará el presente artículo.

Una universidad santandereana, en su preocupación por cualificar procesos integrales, junto con el compromiso de la dirección, reporte de coordinación académica, gestión de bienestar universitario y apoyo de sus docentes investigadores, procedió tanto al estudio de situaciones que ameritaran algún tipo de intervención especial como al diseño e implementación de estrategias didácticas que respondieran a problemáticas latentes. Dichas técnicas son acordes a la situación actual de los estudiantes y en pro de la mejora institucional continua. SDUN es una de las alternativas implementadas.

Dos fueron las categorías principales en torno a la presente investigación, cada una con sus respectivas dimensiones. La primera se denominó capacidad de redacción, y sus dimensiones fueron las siguientes: coherencia, que, según Rodríguez (2013), está relacionada con las relaciones lógico-semánticas entre las partes de una oración y entre una oración y otra; sencillez, sinónimo de claridad y naturalidad (Salazar, 1999); y ortografía, entendida como manera de uniformar el lenguaje para facilitar la comunicación escrita (Jaúregui, 2008). La segunda categoría se conceptualizó como "Semillas de un Nobel", y sus dimensiones fueron: redacción, entendida como una manera de organizar y plasmar el pensamiento (Salazar, 1999); microrrelato, expresión milenaria caracterizada por su brevedad, representación gráfica y diálogo con otros textos (Zaid, 2004); estrategia, que aborda la escritura como herramienta de expresión, recreación y revelación espiritual-epistemológica del pensamiento (Orrantia, 2012), en un sentido plástico en que el arte de pintar con la palabra no es un privilegio de pocos, sino un derecho de todos.

\section{Metodología}

La presente investigación es de paradigma cualitativo, de enfoque hermenéutico. Se hizo la recolección y el análisis de información con base en tres mecanismos: revisión bibliográfica, aplicación de los ejercicios de redacción previstos en la plantilla de actividades para las sesiones con los estudiantes y medición del impacto de la estrategia a través de la entrevista semiestructurada y su aplicación. Durante el desarrollo de la investigación se ejecutaron tres fases: diseño, implementación y evaluación de la estrategia. Sobre estos últimos aspectos se ampliará en el ítem denominado procedimiento.

\section{Tipo de estudio}

Dada la intencionalidad de la presente investigación, se optó por el paradigma de horizonte cualitativo, de enfoque hermenéutico. Principalmente estas fueron las etapas del proceso: recolección, análisis y evaluación de información, a partir de tres mecanismos: revisión bibliográfica, instrumento de observación (plantilla) y entrevista semiestructurada.

\section{Participantes}

Se trabajó con una muestra de 27 estudiantes de las seis carreras profesionales (Psicología, Diseño Gráfico, Ingeniería Industrial, Ingeniería de Sistemas, Contaduría, Administración de Empresas) con las que actualmente cuenta la Universidad de Investigación y Desarrollo (UDI), extensión San Gil. Los criterios de inclusión para la muestra fueron dos: presentar un promedio académico acumulado inferior a 3,5 y que los participantes fueran remitidos por la División de Bienestar Universitario.

\section{Materiales e instrumentos}

Durante el desarrollo de la investigación, el material bibliográfico, los equipos tecnológicos y los implementos de papelería fueron los más empleados. En concreto, textos impresos, computador, proyector, amplificadores de sonido, marcadores, lapiceros y papel. En cuanto a instrumentos de diagnóstico, análisis y evaluación de información, se optó por el concepto de conferencia-laboratorio. Así como, la hoja plantilla, la rejilla de evaluación y la entrevista semiestructurada (ver Anexos 1, 2 y 3 ).

\section{Procedimiento}

Fueron tres las etapas desplegadas durante el proceso. La primera etapa se denomina diseño de la estrategia, y consistió en un ejercicio de sistematización de corte epistemológico y técnico, en el que, partiendo del concepto microrrelato, se procedió a la organización de tres conferencias-laboratorio de redacción. La conferencia, según Robles Garrote (2013), se entiende como discurso interactivo. Por su parte, laboratorio, desde el aporte de López y Tamayo (2012), se concibe como espacio de experimentación textual a partir de indicaciones dadas. Cada intervención tuvo una duración de 60 minutos. Los temas abordados fueron el microrrelato, la ortografía, la coherencia, la sencillez, la autoconfianza, la brevedad, el uso de metáforas y la redacción.

La etapa dos consistió en la intervención pedagógica. En esta, se procedió a la implementación de las tres conferencias-laboratorios de redacción, cada una con duración de 60 minutos. En estas se realizó 
abordaje teórico-práctico en torno al tema-dimensión mencionado. Antes de las intervenciones, cada estudiante recibía una hoja-plantilla en la que, por el lado A (en blanco), componía textos que iban surgiendo a lo largo de las sesiones. De antemano se aclaraba que cada escrito no debía superar las tres líneas. Por el lado B, aparecía una rejilla que posteriormente sería considerada por los docentes encargados de la actividad. Una vez finalizaba el ejercicio, venía la etapa de socialización, en la que cada estudiante era invitado a transcribir el texto que, desde su concepto, había sido el mejor logrado, y posteriormente lo leía en voz alta. Después de cada intervención, se recogían las guías (fueron tres en total por cada estudiante), y posteriormente a cada uno se le entregaba una página del libro de microrrelatos tomado como referente (Lagmanovich, 2006), para su lectura e intercambio. Con la implementación de dichos talleres, se buscó en los estudiantes cualificar su capacidad de redacción, mediante las etapas de sensibilización, planeación, ejecución, revisión y posescritura.

Para la ejecución de la etapa tres, se prestó atención a que la entrevista semiestructurada analizara en qué medida la estrategia "Semillas de un Nobel" (SDUN), así como los subprocesos de revisión textual, contribuían a mejorar las competencias necesarias para una redacción adecuada de tipo académico.

\section{Resultados}

Desde la categoría "Capacidad de redacción", los efectos alcanzados en los estudiantes que hicieron parte de la investigación fueron prometedores. Desde el punto de vista de las dimensiones coherencia, sencillez y ortografía, se reveló una mejora integral aproximada del $33 \%$, en cuanto a redacción, en relación con el nivel inicial de dichos participantes. En la categoría "Semillas de un Nobel", desde las dimensiones redacción, construcción de microrrelatos y "la estrategia", las repercusiones fueron realmente significativas, dado que se alcanzó una mejora aproximada del $60 \%$, en relación con el estado inicial de los participantes.

Sería un gran aporte si se pudiera expresar en porcentajes y cifras tanto las sonrisas como las miradas de aprobación que los estudiantes revelaban en el transcurso de la investigación, al fomentarse en ellos la autovaloración, la autoconfianza y el autoredescubrimiento de sus competencias. Y esto no solo en materia de redacción, sino también en algunos ámbitos más de la vida. Todos los participantes, sin excepción, lograron durante la investigación composiciones textuales dignas de ser publicadas, verdaderas Semillas de un Nobel. Sería muy grato visitar todas las instituciones posibles para demostrar el éxito de la estrategia SDUN y del grado de repercusión que se puede lograr con tan solo media jornada de trabajo. Cuando una persona logra empoderarse, es capaz de superar cualquier expectativa.

\section{Conclusiones}

El objetivo general, analizar el impacto de la estrategia "Semillas de un Nobel" en el mejoramiento de las capacidades de redacción en los estudiantes universitarios, se logró gracias a que los objetivos específicos también se alcanzaron. Estos fueron diseño de estrategia didáctica, implementación de "la estrategia" y evaluación de los cambios en las habilidades de redacción.

A continuación, el análisis del impacto de SDUN desde sus respectivas categorías y dimensiones:

Categoría Capacidad de redacción: En la dimensión coherencia, las relaciones lógico-semánticas son esenciales a la hora de comunicar; para ello se requiere constante lectura de patrones, relectura y autoedición. La sencillez, la claridad, la empatía y la naturalidad deben estar por encima de lo abstracto y artificial. La ortografía, en cuanto apología de la tradición, de la uniformidad, debe respetarse en pro de una comunión y comunicación diáfana del lenguaje.

Categoría Semillas de un Nobel: En la dimensión redacción, se concluyó que dicho ejercicio requiere deliberación; asimismo, coherencia, sencillez, ortografía. Escribir es algo natural; redactar parece ser lo propiamente humano, pues requiere esfuerzo e interacción consciente consigo mismo, con el otro y con el entorno.

Composición de microrrelatos: Se reconoció la importancia de ser concreto, recursivo, creativo y preferir lo esencial por encima de lo accesorio. Asimismo, se adoptó la importancia de la metáfora como manera de hacer plástico lo abstracto, y del diálogo con otros textos como mecanismo de anclaje e inspiración.

"La estrategia": El fomentar la confianza y la autoconfianza motiva en los estudiantes el que la escritura y la capacidad de redacción fluyan. La bandera de SDUN es: "Todos somos escritores por naturaleza: con pasión, disciplina y acompañamiento cualquiera puede llegar a la cima de cualquier texto"; y no solamente a la hora de plasmar en tinta y papel, sino en cualquier ámbito de la vida.

\section{Referencias}

Álvarez, S. \& Mosquera, E. (2014). Normalización del Microtexto. Nuevos desafíos en PLN para el gallego. Universidad de la Coruña.

Arrieta, B. \& Meza (2005). La comprensión lectora y la redacción en estudiantes universitarios. Revista Iberoamericana de Educación, 36(13), 1-10.

Bustamante, L. (2012). Una aproximación al microrrelato 
hispánico: antologías publicadas en España (19902011). Tesis Doctoral. Recuperado de: https:// uvadoc.uva.es/bitstream/handle/10324/1029/ TESIS188120702.pdf;jsessionid $=8$ A8245D1E55DCE3 EAE301DA5ACE7BA24? sequence $=1$

Carrasco, A., Encinas, M. T.., Castro, M. C., \& López, G. (2013). Lectura y escritura académica en la educación media superior y superior. Revista mexicana de investigación educativa, 18(57), 349-354.

Castañeda, L. (2011). Hacia una Erótica del Microtexto. Recuperado de: http://oldversion.razonypalabra.org. $\mathrm{mx} / \mathrm{N} / \mathrm{N} 77-2 / 05$ Castaneda_M77-2.pdf

Castelló, M., Pozo, J. I., \& Pérez-Echeverría, M. P. (2009). Aprender a escribir textos académicos icopistas, escribas, compiladores o escritores (pp. 120-133). Madrid, España: Morata.

CERLALC (2007). La Escritura Creativa en la Escuela. Centro Regional para el Fomento del Libro en América Latina, el Caribe, España y Portugal. Recuperado de: https:// cerlalc.org/wpcontent/uploads/publicaciones/olb/ PUBLICACIONES_OLB_Memorias-Primer-CongresoIberoamericano-de-Libreros_v1_231107.pdf

Espinosa, E., Zambrano, M., Martínez, G., \& Suárez, L. (2005). El impacto del curso de redacción avanzada en la competencia lingüística de comunicación escrita en los estudiantes del Instituto Tecnológico de Estudios Superiores de Monterrey, campus Monterrey. Recuperado de: http://www.mty.itesm. mx/rectoria/dda/rieee/pdf05/39(DHCS).L.SuarezG. Mtz.E.EspinozaM.Zambrano.pdf

Fregoso, G. (2008). Problemas del estudiante universitario con la redacción Un estudio de caso en los niveles de licenciatura y maestría. Revista Universidad EAFIT. 44(149), 9-22 Recuperado de: http://repositorio.cualtos. udg.mx:8080/jspui/bitstream/123456789/161/1/ Problemas\%20del\%20universitario\%20con\%20la\%20 redaccion.pdf

García, G. (2012). Microtextos, Microestilo, Microvoz. Recuperado de: https://thinkcopy.wordpress. com/2012/01/22/microtextos-microestilo-microvoz/

Jaúregui, R. M. (2008). El problema de la ortografía. Educere, 12(42), 625-627. Recuperado de: http:// www.redalyc.org/pdf/356/35614569023.pdf

Lagmanovich, D. (2008). En el territorio de los microtextos. Ínsula: revista de letras y ciencias humanas, (741), 3-5. Recuperado de: https://es.scribd.com/ document/143493602/En-el-territorio-de-losmicrotextos-pdf

Lagmanovich, D. (2006). La extrema brevedad: microrrelatos de una y dos líneas. Espéculo. Revista de estudios literarios, 32, 1-23. Recuperado de: https:// www.biblioteca.org.ar/libros/151584.pdf

López, A. \& Tamayo, O. (2012). Las prácticas de laboratorio en la enseñanza de las ciencias naturales. Revista Latinoamericana de Estudios Educativos (Colombia), 8(1), 145-166. Recuperado de, https://www.redalyc. org/pdf/1341/134129256008.pdf
Ocampo, M. (2016). La "ensalada de cuentos" como estrategia para mejorar la producción de texto cuento en los estudiantes de grado tercero de la institución educativa distrital llano grande J.L. (Doctoral dissertation, Universidad Nacional de Colombia-Sede Bogotá). Recuperado de: http:// bdigital.unal.edu.co/54714/1/2949826555.2016.pdf

Orrantia, M. (2012). La escritura creativa en Colombia. Literatura: teoría, historia, crítica, 14(1), 287-301. Recuperado de: https://revistas.unal.edu.co/index. php/lthc/article/view/30965/39605

Pérez-Abril, M., Rodríguez, A. (2013). ¿Para qué se lee y se escribe en la universidad colombiana? Caracterización de prácticas de lectura y escritura en 17 universidades. Revista de Docencia Universitaria. REDU. 11 (1), 137-160.

Robles Garrote, P. (2013). La conferencia como género monológico: análisis macroestructural en español e italiano. Boletín de filología, 48(1), 127-146. Recuperado de https://scielo.conicyt.cl/scielo.php?pid=S071893032013000100006\&script=sci_arttext

Rodríguez, A. (2013) Cohesión y coherencia: Los conectores. Comunicación oral y escrita. Universidad ICESI. Recuperado de: http://www.icesi.edu.co/blogs/ lenguajevirtual/files/2008/08/los-conectores.PDF

Sabaj, O. (2009). Descubriendo algunos problemas en la redacción de Artículos de Investigación Científica (AIC) de alumnos de postgrado. Revista Signos, 42(69) 107-127 Recuperado de: https://scielo.conicyt.cl/ pdf/signos/v42n69/a06.pdf

Salazar, A. (1999) La redacción: Concepto, características, sus fases. Universidad Metropolitana Unidad Xochimilco. Recuperado de: http://www.posgrado. unam.mx/arquitectura/aspirantes/La_Redaccion.pdf

Sánchez, C. (2005). Los problemas de redacción de los estudiantes costarricenses: Una propuesta de revisión desde la lingüística del texto. Revista de filología y lingüística de la Universidad de Costa Rica, 267295. Recuperado de: https://core.ac.uk/download/ pdf/67717869.pdf

Schmeck, R. R. (1988). An introduction to strategies and styles of learning. In Learning strategies and learning styles (pp. 3-19). Springer, Boston, MA.

Timbal, L. (1993). Escritura Creativa. Madrid: EDAF.

Zaid, G. (2004). Del microtexto al yo. Letras libres, (29), 22-25. Recuperado de: http://www.letraslibres.com/ mexico-espana/del-microtexto-al-yo-0 
Anexo n. 1. Plantilla de Evaluación

\begin{tabular}{|c|c|c|c|c|c|c|c|c|}
\hline \multirow{9}{*}{$\begin{array}{c}\text { Texto mejor } \\
\text { logrado }\end{array}$} & \multirow{6}{*}{$\begin{array}{l}\text { Categoría } \\
\text { Capacidad de } \\
\text { Redacción }\end{array}$} & \multirow{2}{*}{\multicolumn{2}{|c|}{ Dimensión }} & \multicolumn{5}{|c|}{ Puntuación } \\
\hline & & & & 1 & 2 & 3 & 4 & 5 \\
\hline & & \multicolumn{2}{|l|}{ Redacción } & & & & & \\
\hline & & \multirow{3}{*}{$\begin{array}{l}\text { Construcción de } \\
\text { Microrrelato }\end{array}$} & Brevedad & & & & & \\
\hline & & & Metáfora & & & & & \\
\hline & & & Transtextualidad & & & & & \\
\hline & \multirow{3}{*}{$\begin{array}{c}\text { Categoría } \\
\text { Semillas de un Nobel }\end{array}$} & \multicolumn{2}{|l|}{ Coherencia } & & & & & \\
\hline & & \multicolumn{2}{|l|}{ Sencillez } & & & & & \\
\hline & & \multicolumn{2}{|l|}{ Ortografía } & & & & & \\
\hline
\end{tabular}

Fuente: Elaboración Propia.

Anexo n. 2. Plantilla Entrevista Semiestructurada

\begin{tabular}{|c|c|c|c|}
\hline Redacción & ¿Para usted qué es redactar? & $\begin{array}{l}\text { Subraye el texto que considere mejor } \\
\text { redactado } \\
\text { a. La gallina nido perdió su. } \\
\text { b. La gallina perdió su nido. }\end{array}$ & $\begin{array}{l}\text { Redacte un texto con } \\
\text { las siguientes } \\
\text { palabras: } \\
\text { Hambre, tengo, } \\
\text { óyeme, perro, soy, tu. }\end{array}$ \\
\hline Microrrelato & ¿Qué opina del microrrelato? & $\begin{array}{l}\text { Cuál de estos textos es un microrrelato: } \\
\text { a. Cuando duermo con la sábana en el } \\
\text { piso. } \\
\text { b. Cuando despertó el dinosaurio aún } \\
\text { estaba ahí. }\end{array}$ & $\begin{array}{l}\text { Cuáles son algunas } \\
\text { de las características } \\
\text { del microrrelato: } \\
\text { a. Breve } \\
\text { b. Metafórico } \\
\text { c. Hace alusión a } \\
\text { otros textos } \\
\text { d. Todas las } \\
\text { anteriores } \\
\end{array}$ \\
\hline Estrategia & $\begin{array}{l}\text { De } 1 \text { a } 5 \text { (siendo cinco la nota más alta) } \\
\text { cuál es el concepto que usted tiene de sí } \\
\text { mismo (a): } 1,2,3,4,5\end{array}$ & $\begin{array}{l}\text { Cuando usted escribe lo hace por: } \\
\text { a. Gusto } \\
\text { b. Imposición }\end{array}$ & $\begin{array}{l}\text { ¿Cree usted que } \\
\text { podría escribir un } \\
\text { texto que merezca un } \\
\text { aplauso? }\end{array}$ \\
\hline Coherencia & $\begin{array}{l}\text { ¿Cuál es su opinión cuando se dice que a } \\
\text { la hora de escribir cada palabra debe } \\
\text { estar en su lugar? }\end{array}$ & $\begin{array}{l}\text { Ordene la siguiente frase según el orden } \\
\text { que considere: } \\
\text { - Los mujeres y las hombres río y agua } \\
\text { parecen se. }\end{array}$ & $\begin{array}{l}\text { ¿Qué puede pasar } \\
\text { ante un escrito que } \\
\text { carece de orden y } \\
\text { lógica? }\end{array}$ \\
\hline Sencillez & $\begin{array}{l}\text { ¿A la hora de escribir prefiere ser sencillo } \\
\text { o rebuscado? }\end{array}$ & $\begin{array}{l}\text { Subraye el término que emplearía como } \\
\text { sinónimo de opinión: cosmovisión, } \\
\text { racionalidad, mirada. }\end{array}$ & $\begin{array}{l}\text { Subraye cuál es el } \\
\text { propósito del } \\
\text { lenguaje: } \\
\text { complejizar, alardear, } \\
\text { comunicar. }\end{array}$ \\
\hline Ortografía & ¿Cuál es su opinión de la ortografía? & $\begin{array}{l}\text { ¿Qué diría si su pareja al terminar la cita le } \\
\text { escribe: "Te hamaré siempre"? }\end{array}$ & $\begin{array}{l}\text { Cuál es la palabra } \\
\text { que más se le } \\
\text { dificulta escribir } \\
\text { correctamente. }\end{array}$ \\
\hline
\end{tabular}

Fuente: Autores. 
Anexo $n .^{\circ}$ 3. Evolución del proceso en la etapa inicial y de evaluación

\begin{tabular}{|c|c|c|c|}
\hline & & Primera etapa del Proceso & Etapa de evolución del Proceso \\
\hline & Dimensión Coherencia & $\begin{array}{l}\text { Inicialmente los estudiantes } \\
\text { estaban en un nivel básico de } \\
\text { puntuación } 2\end{array}$ & $\begin{array}{l}\text { Las relaciones lógico-semánticas son esenciales } \\
\text { a la hora de comunicar; para ello se requiere } \\
\text { constante lectura de patrones, relectura y } \\
\text { autoedición. En una puntuación } 4\end{array}$ \\
\hline \multirow[t]{3}{*}{$\begin{array}{c}\text { Categoría } \\
\text { Capacidad de Redacción }\end{array}$} & Dimensión Sencillez & $\begin{array}{l}\text { En esta dimensión una puntuación } \\
\text { de } 3\end{array}$ & $\begin{array}{l}\text { La claridad, la empatía y la naturalidad debe } \\
\text { estar por encima de lo abstracto y artificial. En } \\
\text { una puntuación } 4 \text {. }\end{array}$ \\
\hline & Dimensión Ortografía & $\begin{array}{l}\text { En esta dimensión una puntuación } \\
\text { inicial de } 1\end{array}$ & $\begin{array}{l}\text { La ortografía como apología a la tradición, a la } \\
\text { uniformidad debe respetarse en pro de una } \\
\text { comunión y comunicación diáfana del lenguaje. } \\
\text { En una puntuación } 3 \text {. }\end{array}$ \\
\hline & Dimensión Redacción & $\begin{array}{l}\text { En este apartado los participantes } \\
\text { estaban a una puntuación de } 2\end{array}$ & $\begin{array}{l}\text { Dicho ejercicio requiere deliberación, asimismo } \\
\text { coherencia, sencillez, ortografía. Finalmente se } \\
\text { determinó una puntuación de } 4 \text {. }\end{array}$ \\
\hline \multirow[t]{2}{*}{$\begin{array}{l}\text { Categoría } \\
\text { Semillas de un Nobel }\end{array}$} & $\begin{array}{l}\text { Dimensión construcción } \\
\text { de Microrrelatos }\end{array}$ & $\begin{array}{l}\text { Al inicio los estudiantes solo } \\
\text { presentaban una puntuación de } 1\end{array}$ & $\begin{array}{l}\text { Concreto, recursivo, creativo, y preferir lo } \\
\text { esencial por encima de lo accesorio; así mismo } \\
\text { se adoptó la importancia de la metáfora. Este } \\
\text { ítem fue el que presentó mejor rendimiento } \\
\text { luego de la estrategia, con una puntuación de } 5 .\end{array}$ \\
\hline & Dimensión La Estrategia & $\begin{array}{l}\text { Al inicio los estudiantes } \\
\text { presentaron una puntuación de } 1\end{array}$ & $\begin{array}{l}\text { La estrategia consiste en el fomentar la } \\
\text { confianza y la autoconfianza en los estudiantes, } \\
\text { lo que permite que la escritura y la capacidad de } \\
\text { redacción fluya. Se logró una puntuación de } 4 \text { al } \\
\text { final. }\end{array}$ \\
\hline
\end{tabular}

Fuente: Autores. 